\title{
Microneedles in diagnostic, treatment and theranostics: An advancement in minimally-invasive delivery system
}

\author{
Ragini Amarnani ${ }^{1} \cdot$ Pravin Shende $^{1}$
}

Accepted: 23 November 2021 / Published online: 8 December 2021

(c) The Author(s), under exclusive licence to Springer Science+Business Media, LLC, part of Springer Nature 2021

\begin{abstract}
Microneedle $(\mathrm{MN})$ technology plays an important role in biomedical engineering for their less intrusive access to the skin due to minimally or painless penetration, enhancement of drug permeability, improvement of detectability of biomolecules in the epidermal and dermal layers with therapeutic efficacy and safety. Furthermore, MNs possess some major disadvantages like difficulty in scale-up technique, variation in drug delivery pattern with respect to external environment of skin, blockage of arrays due to dermal tissues, induction of inflammation or allergy at the site of administration and restriction of dosing range based on the size of active. Additionally, microneedle acts as a transdermal theranostic device for monitoring the physiological parameters in clinical studies. The investigation of drug transfer mechanisms through microneedles includes coat and poke, poke and flow, poke and patch and poke and release method. This review article discusses different categories of microneedles with fabrication methods such as photolithography, laser cutting, 3D printing, etc. in therapeutic applications for treating cancer, diabetes, arthritis, obesity, neurological disorders, and glaucoma. Biosensing devices based on microneedles may detect target analytes directly in the interstitial fluid by penetrating the stratum corneum of the skin and thus microneedles-based devices can be considered as a single tool in diagnostic sensing and therapeutic administration of drugs inside the body. Moreover, the clinical status and commercial availability of microneedle devices are discussed in this review article to offer new insights to researchers and scientists. Continuous monitoring particularly for the determination of blood glucose concentration is one of the most important requirements for the development of next-generation healthcare devices. The aim of this review article focuses mainly on the theranostic applications of microneedles in various medical conditions such as malaria, glaucoma, cancer, etc.
\end{abstract}

Keywords Microneedle $\cdot$ Fabrication $\cdot$ Drug delivery $\cdot$ Diagnosis $\cdot$ Regulatory requirement

\section{Introduction}

The skin is the largest part of the body, it accounts for $16 \%$ of total body weight and consists of the epidermis, dermis, and hypodermis of which the uppermost skin layer stratum corneum allows permeation of only low molecular weight drugs $(<500 \mathrm{Da})$ such as clotrimazole, terbinafine, etc. (Babity et al. 2018). Moreover, it permits only lipophilic actives with low melting points to limit the drawbacks of conventional transdermal drug delivery systems (Moreira et al. 2019). Microneedles (MNs) are insignificant invasive devices used to sense

\section{Pravin Shende}

Shobhaben Pratapbhai Patel School of Pharmacy and Technology Management, SVKM'S NMIMS, V. L. Mehta Road, Vile Parle (W), Mumbai, India and transport active pharmaceutical ingredient (API) across stratum corneum by penetrating the epidermal layer of the skin without puncturing the nerves and causing extreme pain (Zhang et al. 2020). The administration of active molecules via skin the is mostly carried out by topical cream, transdermal patch, hypodermic needles, etc. (Waghule et al. n.d.). Transdermal devices are adhesive tools applied to the skin for the administration of therapeutics via the stratum corneum, however, limited bioavailability across the multiple layers of skin restricted the applications in healthcare (Rastogi and Yadav 2012). Table 1 compares different dosage forms such as topical, parenteral, transdermal and trans-epidermal. The recent innovations in microelectronics and micromachinery in combination with the precision of modern computers enable better treatment outcomes for chronic and lifethreatening conditions such as cancer and diabetes (Khare 
Table 1 Comparison of MNs over different dosage forms

\begin{tabular}{|c|c|c|c|c|}
\hline Description & Topical cream & Parenteral injection & Transdermal patch & Trans-epidermal \\
\hline $\begin{array}{l}\text { The molecular weight of } \\
\text { drugs }\end{array}$ & $\begin{array}{l}\text { Insufficient for } \\
\text { high } \\
\text { molecular } \\
\text { weight drugs }\end{array}$ & $\begin{array}{l}\text { Insufficient for high molecular } \\
\text { weight drugs }\end{array}$ & $\begin{array}{l}\text { Insufficient for high molecular } \\
\text { weight and lipophilic drugs }\end{array}$ & $\begin{array}{l}\text { Serves as a potential delivery } \\
\text { route for both low and high } \\
\text { molecular weight drugs }\end{array}$ \\
\hline Bioavailability & $10-20 \%$ (poor) & $\begin{array}{l}100 \% \\
\text { (sufficient) }\end{array}$ & Insufficient & Sufficient \\
\hline Onset of action & Slow & Faster & Slow & Fast \\
\hline Pain & Painless & Painful & Painless & Painless \\
\hline Self-administration & Possible & Impossible & Possible & Possible \\
\hline Patient compliance & Less & Less & Better & Better \\
\hline Mechanism of drug delivery & $\begin{array}{l}\text { Pores act as a } \\
\text { drug carrier } \\
\text { system }\end{array}$ & $\begin{array}{l}\text { Drug enters directly into the } \\
\text { dermis }\end{array}$ & $\begin{array}{l}\text { Poor permeability across } \\
\text { stratum corneum }\end{array}$ & $\begin{array}{l}\text { Drug directly enters the } \\
\text { epidermis, bypasses the } \\
\text { stratum corneum and } \\
\text { increases the permeability }\end{array}$ \\
\hline Microneedle size & - & $1.5-4 \mathrm{~mm}$ long needle & $10-40 \mu \mathrm{m}$ long needle & $150-1500 \mu \mathrm{m}$ long needle \\
\hline
\end{tabular}

and Shende 2021). The three-dimensional micro-sized structures with extremely sharp needle-like projections penetrate through the skin and enable drug transport at the targeted site (Sharma 2018). Figure 1 describes the advantages of MNs in drug delivery.

The MNs are small enough to efficiently penetrate across the body's invasive channels to offer excellent drug permeability for large size molecules (Waghule et al. n.d.). MNs are present in different sizes and shapes like $150-1500 \mu \mathrm{m}$ long or $50-250 \mu \mathrm{m}$ in base-width, $1-25 \mu \mathrm{m}$ in tip diameter, heights of $50-900 \mathrm{~cm} 2$, up to $2000 \mathrm{~cm} 2$ depending upon the material used for fabrication and the requirement for the type of MN. Geometry of MNs is of significant importance as it encompasses greater strength and higher ability to pierce the skin for superior drug penetration (Xie et al. 2020).

\section{Classification of MNs-based on theranostics}

MNs can be classified into four types based on the drug delivery methods: solid, coated, hollow and dissolving MNs wherein, solid MNs are used as a skin pre-treatment to create large pores for transport and allowing the drug to slowly diffuse through the pores. Solid MNs are usually made up of glass. where, the base layer provides mechanical support and the remaining layers containing the drug deliver therapeutic effect (Cárcamo-martínez et al. 2021). It shows the advantage of preventing the infection at the site of action with lower therapeutic effect.

Coated MNs contain a drug layer, which allow immediate removal from the skin after releasing the drug from the coating membrane. A recent study demonstrated the

Fig. 1 Advantages of MNs

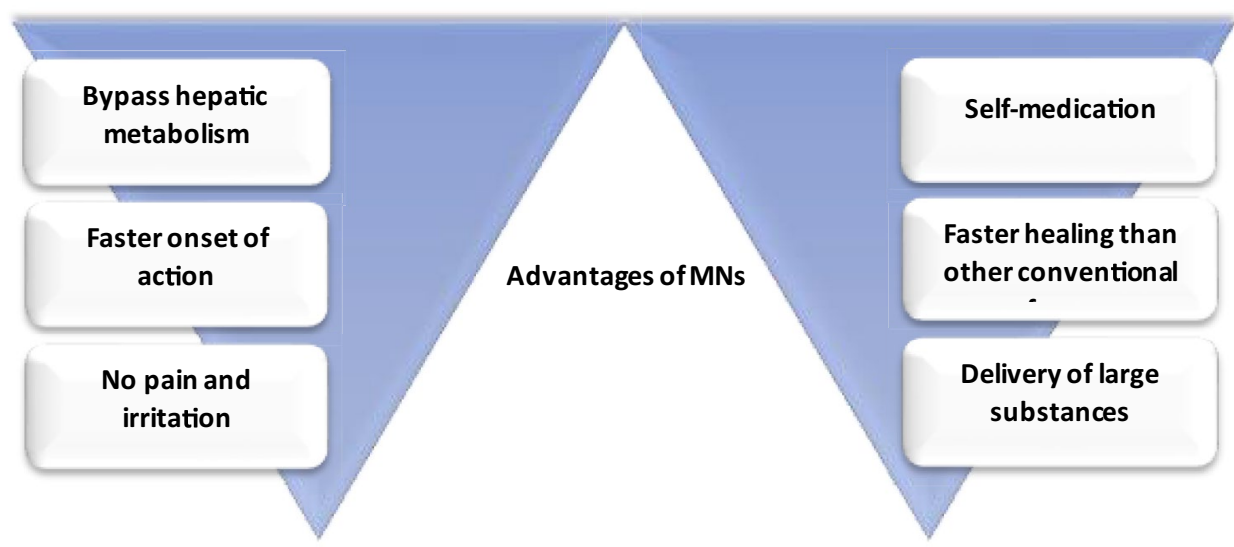


administration of desmopressin via coated MNs across guinea pig's hairless skin for 5 to $15 \mathrm{~min}$ as compared to conventional delivery methods (Cormier et al. 2004).The coating layer and size of the MNs determine the amount of drug to be loaded in the formulation wherein the needles showed the ability to administer water-soluble and water-insoluble drugs and at the same time extending their application in the transportation of drug and as a biosensing tool. Thus, MNs acted as a biosensor when the enzymes were loaded in coated layer for the detection of biomarkers or cells (Bariya et al. 2012). The dissolution time of a drug was enhanced by coating the MNs, for example, the MN was coated with gold to improve biocompatibility and robustness (Donnelly et al. 2010). The halfway insertion of sodium fluorescein-coated MNs through the cornea of rabbit eyes for $2 \mathrm{~min}$ increased intraocular fluorescein concentration instantly after $1 \mathrm{~min}$, followed by a steady increase in peak concentration $(3 \mathrm{~h}$ ) and later, progressive reduction to baseline within $24 \mathrm{~h}$ (Jiang et al. 2007).

Hollow MN are made of ceramics, metal, silicon, and glass, with an empty hollow within each needle and a bore on the needle tip, as previously described. As a result, medication solutions in microvolumes can be injected into the skin. Chemical compounds, proteins, vaccines, and oligonucleotides may all be administered in the skin using this form of MN, which has a greater drug delivery capability than solid, coated, and dissolving MN arrays. They have a high rigidity because to the materials utilized in their construction. Blockage of the bore of needle tips by skin tissue during insertion has been described as a restriction. This problem can be overcome by retracting the $\mathrm{MN}$ array and/ or positioning the bore on the MN side (Cárcamo-Martínez et al. 2021). Furthermore, for the construction of hollow $\mathrm{MN}$ arrays, precise manufacturing processes like as lithography, etching, microelectromechanical systems (MEMS), and, more recently, 3D printing are required, all of which are discussed in the next section (Bhatnagar et al. 2019). This is widely used to analyze the blood and ISF for the detection of ions or glucose in the blood or biological fluids and this leads to the delivery of insulin or vaccine are more prominent action. The hollow MNs possess one major drawback wherein the needles are mostly clogged while inserting into the skin (Cárcamo-martínez et al. 2021). Hollow MNs are used to transport liquids containing soluble compounds such as poly-lactic acid nanospheres and microparticles into the sclera with infusion volumes ranging from 10 to $35 \mu \mathrm{L}$ (Shende and Salunke 2019).

Dissolving MNs completely dissolve or degrade in the skin interstitial fluids and allow the release of encapsulated active at the desirable site (Gupta and Yadav 2019). The major benefit is higher doses transferred into skin and simple manufacturing using molding procedures at ambient conditions makes industrial mass production convenient (Halder et al. 2020). MN arrays are fabricated using a variety of materials, including polyvinyl alcohol (PVA), dextran, polyvinylpyrrolidone (PVP), carboxymethyl cellulose (CMC) and sugars for long-term therapy and patient compliance due to the self-dissolving property of polymer (Van Der Maaden et al. 2012). MNs made of maltose disintegrate in human skin in less than 10 min after insertion but require temperatures above $100{ }^{\circ} \mathrm{C}$ to undergo a transition into the glassy phase (Tabassum et al. 2011). In a research study nanoparticulate dissolvable microneedles proved to be an alternative for increasing the immunopotentiation of tetanus toxoid (Waghchaure et al. 2021).

\section{Mechanisms involved in diagnosis and treatment}

The MNs temporarily disrupt the superficial skin layer via insertion of the needles into the epidermal region, further enabling the drug to reach the systemic circulation. The four mechanisms for systemic drug circulation using MNs is as follows:

\subsection{Poke and patch}

In this mechanism, transdermal patches or prototypes are used to enable MNs to pierce through skin and are accompanied by the application of electric field, sonophoresis, or iontophoresis for enhancing the drug diffusion (Tucak et al. 2020). This approach was formerly applied using solid MNs that form the channels into the skin for the delivery of a drugusing simple diffusion mechanism (Guillot et al. 2020). Solid metal MNs of size range $500 \mu \mathrm{m}$ proved to be safe and effective in administration of insulin solution to experimentallyinduced diabetes rats for $4 \mathrm{~h}$, reduces glucose levels by nearly $80 \%$ due to the microchannels formed into the skin (Zhou et al. 2010).

\subsection{Coat and poke}

In contrast to the prior method, this mechanism involves the application of a drug coat onto the surface of MNs through which the drug is continuously diffused into the skin and reaches the deeper epidermal layers (Barry 2001). In recent research, MNs were used to deliver ovalbumin, a model protein antigen coated on the needle surface with a length of $400-600 \mu \mathrm{m}$. A dry-film coating of ovalbumin antigen was applied to MNs and injected into the skin of hairless guinea pigs and the results demonstrated that coated MNs are more efficient (50\%) than IM or SC route in transporting the drug due to the small size of the MN tip which offers larger surface area (Widera et al. 2006). 


\subsection{Poke and flow}

This method was designed to transport the medication into the skin via hollow MNs mimicking hypodermic injections. A research study conducted with a single hollow MN for the delivery of local anesthetic, lidocaine exhibited excellent pain relief using the Visual Analogue Scale (VAS) with a high patient satisfaction rate. Furthermore, $77 \%$ of the participating patients displayed a better experience with MNs while $80 \%$ stated MNs were barely painful (Zhang et al. 2012).

\subsection{Poke and release}

MN tips are comprised of a biodegradable polymer, that dissolves into the skin to release the drug in a controlled manner. Furthermore, unlike the "poke and patch" method, MNs stay on the skin after application until the drug is released. After absorption into the interstitial fluid of skin, the swelling behavior of MNs produces jelly-like substances known as "bulbs" to activate the primary process in the treatment of diseases like cancer, malaria, hypertension, etc. (Lee et al. 2020). For instance, in a research study hydrogel, MNs were formed and inserted inside the skin, the drug release was followed by swelling of the MNs due to interstitial fluid uptake (Donnelly et al. 2012). Collection of blood sample using hypodermic needles is a painful experience while the interstitial fluid (ISF) is easy to withdraw using MNs serves as a good candidate for biosample analysis due to direct contact with the plasma and contain chemicals and proteins that surround dermal cells (Tuan-Mahmood et al. 2013). Furthermore, ISF maintains the outflow of solutes from the capillaries with the help of hydrostatic and osmotic pressures. The use of MNs for diagnosis proves to be efficient due to reduction in penetration depth from $400-900 \mu \mathrm{m}$ to $50-150 \mu \mathrm{m}$ and thus can be applied for glucose and cholesterol monitoring (Liu et al. 2020). Figure 2 depicts the mechanism of drug release in treatment and diagnosis via MNs.

\section{Fabrication techniques in the development of $\mathrm{MN}$-based theranostics}

Micro-Electro-Mechanical System (MEMS) is a technology that permits accurate uniform manufacturing of MNs to build precise devices and is the most promising method for fabricating ideal MN design (Singh et al. 2013). The foremost MNs were prepared by etching arrays of micronsized needles into silicon using typical microfabrication processes and developed through a variety of materials including ceramic, polydimethylsiloxane (PDMS), glass, polymers and dextrin as well as metals like stainless steel and titanium (Krieger et al. 2019). Among these, silicon is the most prominent material used in this technique due to its cost-effectiveness and easy fabricating procedure. The application of MEMS technology in biological fields (known as biomes) lead to the emergence of applications in monitoring, treatment, DNA sequencing devices, biosensors, and drug delivery (Gowers et al. 2019). Various other methods used for the manufacturing of MNs include cutting, laser cutting, lithography, photolithography, 3D printing, micro-molding, etc. (Liu et al. 2020).
Fig. 2 Drug release mechanisms: A poke and flow: drug transport by diffusion or pressure-driven, $\mathbf{B}$ coat and poke: after crossing the stratum corneum drug releases, $\mathbf{C}$ poke and patch: the drug is transported via passive diffusion by creating pores and $\mathbf{D}$ poke and release: the drug release occurs by the dissolution process
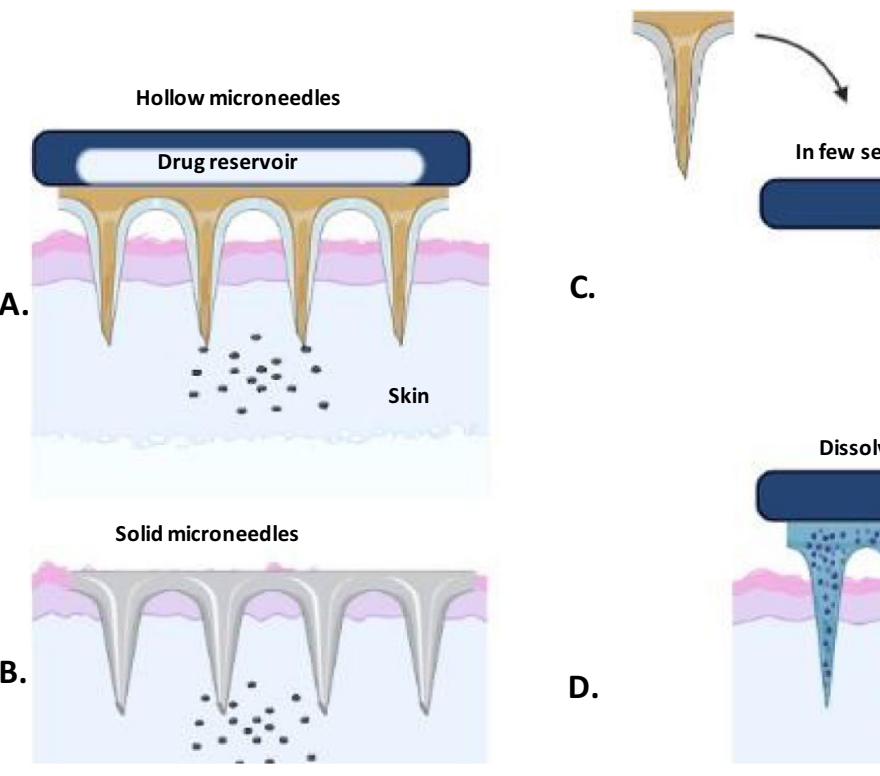

c.

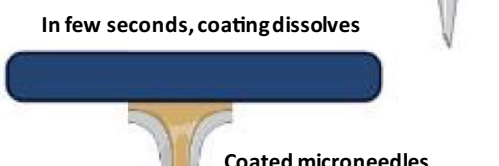

D.
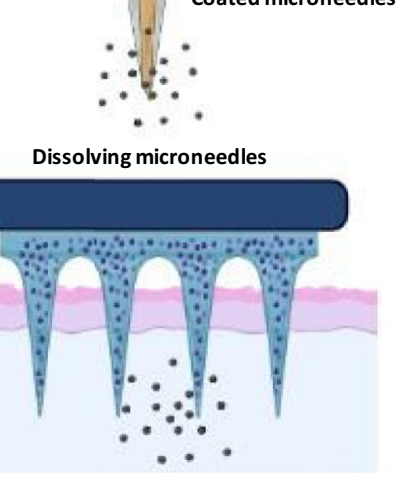


\subsection{Cutting}

Stainless steel sheets are designed by cutting solid MNs with a laser to fabricate the desired size and shape with the help of CAD software. This manufacturing method of MNs using hard material like silicon is not frequently used as the metal blades of the equipment deteriorate the product. MNs are made by either cutting metal with an infrared laser and manually bending the MN structure or by electropolishing which facilitates the reduction of MN insertion time as well as drug permeation (Larrañeta et al. 2016a, b). The beam follows the needle's shape, wherein the $\mathrm{MN}$ is cleaned with hot water and bent at $90^{\circ}$ followed by electropolishing the MNs with compressed air to decrease the width of the $\mathrm{MN}$ or improve the tips of MNs. Thus this technique uses the laser for cutting, known as 3D laser cutting (Yang et al. 2019).

\subsection{Etching}

This method is used for cutting unprotected sections of solid using a powerful liquid (acid) or a physical method to form a design. The different etching techniques include dry and wet etching, the application is dependent on the construction material and the type of MNs to be manufactured (Bariya et al. 2012). The wet etching technique utilizes a liquid to remove the layers from the surface of the MN to manufacture the desired shape whereas, in dry etching, gas is used, however, it is an expensive technique and requires processes like vapor phase or plasma etcher. In a research study, MNs were fabricated using a chromium masking material through a dry reactive ion etching process (Donnelly et al. 2010).

\subsection{Photolithography}

In microelectronic technology, photolithographic techniques require spin-coating a liquid photosensitive polymer termed photoresist onto a substrate, which is then hardened by a heat process. The photoresist is then subjected to UV radiation through a mask, replicating the design of the building to be built. Generally a design is created (termed as the master pattern) and considered as a reference to copy onto a thin substrate usually a silicon wafer to generate a pattern of holes (Dardano et al. 2015). It utilizes a radiation source (UV-Visible light) to transmit a designed shape from the photomask to the substrate which is generally pre-coated with a photosensitive material for manufacturing hollow MNs (Tucak et al. 2020).

\subsection{Micro molding}

Either nano- or micro-size molds are used to create MNs consisting of silicon material wherein, the hot polymeric solution is poured, centrifuged, dried and cooled to form MNs. The various steps of molding are:

a. The molten liquid material is cast into the mold.

b. The air bubbles are removed with the help of centrifugation.

c. Further, the MNs are set aside for solidification and removed from the molds.

This technique is very convenient for the mass production of MNs used in manufacturing sensitive drug loading MNs, however, it is prone to problems such as the presence of air bubbles and high viscosity. To overcome such limitations, an automated spray technique was developed with the molding process and is popular in fabricating ceramic MNs wherein both biodegradable and synthetic materials are used (Agrawal et al. 2018).

\subsection{D printing}

The enormous challenges of precisely manufacturing MNs on a microscale have slowed their commercialization and clinical applications due to their fabricating cost and processes. Thus, the 3D printing technique enables the fabrication of MNs not only in a single (print and fill) step but also in personal customization is possible. This technique is based on a computer-aided drug development model which selectively places the material, layer-by-layer to produce MNs with varied shapes and geometry (Yang et al. 2021). Various 3D printing technologies such as binder jetting, powder bed fusion, material extrusion, sheet lamination and photopolymerization propose to form versatile MNs for diverse applications in the health care industry (Yang et al. 2019). This technique enables the fabrication of both bio-inspired and artificial MNs for minimally invasive transdermal delivery (Krieger et al. 2019). Table 2 depicts the classification of MNs-based on the method of fabrication for the diagnosis of various diseases in the health care industry.

\section{Applications}

\subsection{Immunization}

MNs can deliver high molecular weight protein (up top to $200 \mathrm{Da}$ ) and vaccines across the skin. The dendritic cells with antigenic fragments present in the upper layer of skin activate the immune response by sending a message to $\mathrm{T}$ cells which are further bound to the major histocompatibility complex (MHC complex) for the induction of CD4+ and CD8+ cytotoxic cells (Levin et al. 2015). Another route for activation of immune response is via Langerhans cells to activate the lymphocytes for the formation 
Table 2 Classification of microneedles based on the method of fabrication for diagnosis

\begin{tabular}{llll}
\hline Microneedle type & Fabrication method & Drug & Reference \\
\hline Solid MN & $\begin{array}{c}\text { Micro-molding, etching, 3D } \\
\text { printing, lithography }\end{array}$ & Amantadine, pramipexole & (Hoang et al. 2015) \\
& $\begin{array}{l}\text { Micro-molding, photolithography } \\
\text { Coated MN }\end{array}$ & Lidocaine & (Zhang et al. 2015) \\
Hollow MN & Photolithography, 3D printing & Etanercept, phenylephrine & (Cao et al. 2019) \\
& & & (Jun et al. 2015) \\
\hline
\end{tabular}

of antibodies if an antigen enters the body (Halder et al. 2020). Penetration of the stratum corneum layer and deposition of the NP formulation into these immune cell-rich skin layers promote $\mathrm{MN}$ technologies for the delivery of therapeutic medicines, including vaccinations, to these layers of the skin. Polymeric dissolving MNs are effective for the delivery of vaccines as they dissolve rapidly in the skin without leaving harp wastage (Zaric et al. 2013). The MNs containing antibodies (immunoglobulin Y) efficient for MERS-cCoVS1 and SARS-cov-2 spike proteins were developed wherein MERS-cov-S1 vaccinations administered by MNs elicited strong humoral responses than parenteral route (Kim et al. 2020). For administration of the inactivated poliovirus vaccine, hollow MNs made of hydrofluoric acid-bonded silica capillaries up to the insertion depth of $300 \mu \mathrm{m}$ were used to immunize rats to deliver the drug efficiently into the skin (Van Der Maaden et al. 2014).

\subsection{MNs for drug delivery to the brain}

The blood vessels that carry oxygen to the central nervous system are made up of specialized cells called tight junctions that allow the passage of only lipophilic drugs $(<400 \mathrm{Da})$ and are responsible for the regulation of ions and chemicals. This physiological barrier also known as the blood-brain barrier is responsible for interrupting drug delivery for the treatment of brain tumors, Parkinsonism, Alzheimer's disease, etc. (Agrawal et al. 2018). The conventional methods for drug delivery to the brain include intracerebral injections, intrathecal injections, intraperitoneal injections, etc. which are associated with pain, infection, and inflammation at the site of injection. In recent research, the researchers developed MNs in the $\mathrm{n}$ size range of $25-25,000 \mu \mathrm{m}$ for delivery of biocompatible calcium phosphate nanoparticles for the treatment of cancer. This demonstrated the macrophages engulfed the drug in peripheral circulation and elicit the therapeutic action in the brain (Wong et al. 2010). Another research study prepared and evaluated hydrogel MN in-vitro and in-vivo for transport of donepezil hydrochloride in treating Alzheimer's disease which prevents the occurrence of major GI side effect dysphagia caused by oral delivery (Kearney et al. 2016).

\subsection{MNs for disease treatment}

\subsubsection{Cancer}

The use of chemotherapy or surgery in cancer treatment causes various side effects (toxicity, alopecia) while in nanoparticle-based anticancer therapy, only $0.7 \%$ of the drug reaches the tumor site. The antigens enter the body via MNs and trigger the systemic response that activates the immune cells causing the death of cancer cells (Dardano et al. 2021). The MNs enable the combination of drugs to enter deeper tissues minimizing the leakage and side effects resulting in enhancement of antitumor effect. In a research study 5- amino levulinate-dissolving MN patches were produced utilizing a polymer sodium hyaluronate for tumor therapy. The prepared MNs were determined to be safe and efficient to penetrate the SC, followed by drug disintegration and release. Furthermore, when compared to injectable formulation, tip-loaded MNs of 5-aminolevulinic depicted a higher melanoma inhibitory rate $(97 \%)$ than parenteral formulation (66\%) due to the characteristics of MNs, delivering the drug at the right time and amount by forming microchannels in the kin and thus increasing the bioavailability of drug (Kim et al. 2016). The researchers developed dissolving MN using polyvinyl alcohol (PVA) and polyvinylpyrrolidone (PVP) for doxorubicin and docetaxel co-delivery in breast cancer treatment as combination of photothermal and chemotherapy together aids increasing efficacy of cancer treatment than monotherapy (Venuganti et al. n.d.).

\subsubsection{Diabetes}

Diabetes is a chronic condition faced by $9.3 \%$ of the global adult population (20-79 years) and characterized by high blood glucose levels wherein, most cases require parenteral insulin administration leading to poor patient compliance (Halder et al. 2020). Though oral delivery is desirable, it results in degradation of insulin in the GI tract which can be overcome by percutaneous delivery using MNs. Delivery of insulin via the transdermal route serves as a challenge due to the large size of the insulin molecule (5808 Da), thus, the MNs-based system was developed to deliver the antidiabetic drugs (metformin) in the systemic circulation by creating pores (Zhou et al. 2010). Administration of metformin via 
hydrogel MN patch exhibited controlled release for over $24 \mathrm{~h}$, whereas when given orally the therapeutic effect was limited to $4 \mathrm{~h}$ (Gupta and Felner n.d.). MNs are also useful for monitoring blood glucose wherein for extracting the ISF the penetration depth of MNs should be 50-150 $\mu \mathrm{m}$. Glucose oxidase is the enzyme responsible for the conversion of glucose to gluconic acid with the help of oxygen (Chang et al. 2017). In the case of hypoxia or increased level of glucose, the oleophilic 2-nitroimidazole converts into hydrophilic 2-aminoimidazole and dissociates hyaluronic acid vesicles and results in insulin release. Thus, glucose oxidase enzyme is used in the formulation of MNs along with insulin which acts as a biosensor and releases insulin in hypoxia condition (Yu et al. 2016).

\subsubsection{Arthritis}

Rheumatoid arthritis (RA) is a chronic, autoimmune, inflammatory condition associated with joint pain, drugs such as abatacept, adalimumab, allopurinol, aspirin, methotrexate, etc. are used to alleviate the pain (Bullock et al. 2018). Etanercept is usually given as a subcutaneous injection, resulting in poor patient compliance, adverse effects, and infection risk. A research study developed etanercept-loaded (TNF $\alpha$ inhibitor) dissolvable MN crosslinked with hyaluronic acid in the size range of $50-900 \mu \mathrm{m}$ to deliver the drug transepidermal which improves biocompatibility and possess high anti-inflammatory efficacy in mice (Cao et al. 2019). In a recent study, a methotrexate-loaded MN array containing PLGA microspheres of folic acid was formulated to treat RA to increase the bioavailability of methotrexate and enable dose reduction (Shende and Salunke 2019). DEK is the protein responsible for the formation of neutrophil in RA. So, by blocking this protein by aptamer DTA6 proves to be efficient for rheumatoid treatment but, this DTA is prone to nuclear degradation and renal clearance. Therefore, the formation of hydrogel MN for delivery of DTA6 demonstrated the increase in stability of aptamer up to $72 \mathrm{~h}$ in $90 \%$ mice and thus protects the joints/bones from erosion in collagen-induced arthritis (Cao et al. 2021). Figure 3 depicts the hydrogel $\mathrm{MN}$ drug delivery to inflamed joint for treatment of collagen-induced arthritis.

\subsubsection{Obesity}

Excessive accumulation of fats in these tissues leads to obesity and drugs such as lipase inhibitor (orlistat), appetite suppressor (dexfenfluramine) are used in the treatment of obesity (Zhang et al. 2018). Caffeine and catechin extracted from tea act synergistically to reduce obesity, however, topical delivery in crystalline form inhibits its optimum efficacy in reducing obesity (Zheng et al. 2004). Delivery of caffeine in combination with hyaluronic acid in form of $\mathrm{MN}$

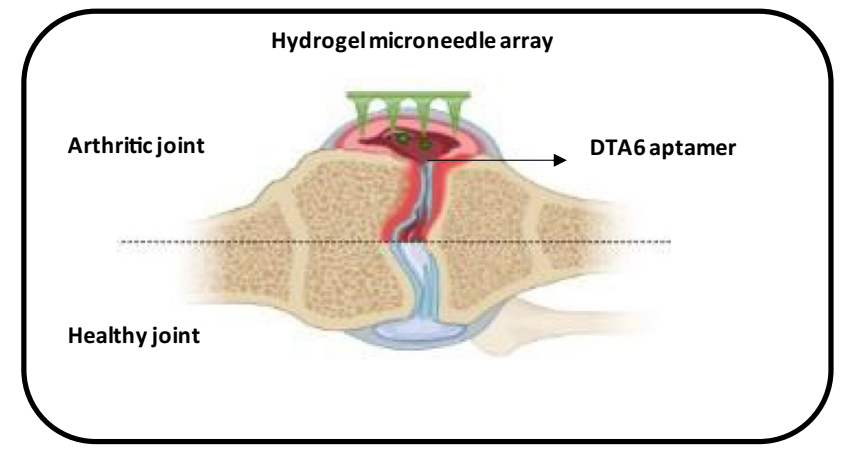

Fig. 3 Hydrogel MN drug delivery to inflamed joint for treatment of collagen-induced arthritis

patch promotes solubility of caffeine in the formulation and prevents crystal formation which is more effective than the conventional method (Dangol et al. 2017). Two types of adipose tissue i.e., white adipose tissue (stores fats) and brown adipose tissue (expenditure of fats by producing energy) are present in subcutaneous tissues of mammals. Another research study developed MN of rosiglitazone (browning agent) that converts WAT into BAT for treatment against obesity due to the presence of $\beta-3$ adrenoreceptor agonist that releases the medication slowly (the due to long dissolving nature of MN) (Zhang et al. 2017).

\subsubsection{Neurological disorders}

MNs are so used in delivery of ACH inhibitors to treat Alzheimer's disease caused due to a reduction in the level of acetylcholine. Donepezil is considered a first-line drug for the treatment of Alzheimer's disease however; its oral administration leads to gastrointestinal side effects like nausea and vomiting. Furthermore, oral administration in geriatric patients is difficult, thus, epidermal delivery can prove to be more efficient but due to its hydrophilicity and high molecular weight (415.96), the drug is unable to achieve sufficient permeation (Nguyen et al. 2017). A study demonstrated pretreatment of the skin by solid MNs loaded with lidocaine produced anesthesia within $30 \mathrm{~min}$ while topical $4 \%$ lidocaine cream showed the therapeutic effect in $60 \mathrm{~min}$ (Gupta et al. 2012). Chronic neuropathic pain is caused by trauma and inflammation is a major problem for $50 \%$ of the population. When the injury occurs, it triggers calcitonin gene-related peptide (CGRP), released by nociceptive sensory neurons leading to thermal hyperalgesia, neuropathic pain and it can be treated by blocking the CGRP neuropeptide. Local anesthetics, NSAIDs, and antidepressants are used to treat this disorder but possess certain side effects like slow heart rate, tremors, etc. Certain local anesthetics (lidocaine, bupivacaine, etc.) were given transdermally, however, chronic application caused numbness in the skeletal muscles due to blockade of sodium channels. CGRP8-37 an 
anti-CGRP peptide was delivered through dissolving MNs patches into the affected area avoiding systemic exposure and side effects (Xie et al. 2017).

\subsubsection{Ophthalmic}

Treatment of ophthalmic disorders is highly challenging due to inaccessibility to ocular tissues, poor bioavailability offered by topical drug application, low formulation retention time, etc. Furthermore, the presence of various anterior and posterior barriers such as the cornea, blood-aqueous barrier, tear film, etc. and thus requires a higher concentration of drugs that can cause toxic effects (Thakur Singh et al. 2017). MNs up to $200 \mu \mathrm{m}$ in size range cross all the ocular barriers and are administered at the site of the affected area (Gupta and Yadav 2019). MNs were formed to administer the drug in the CS region (between the sclera and choroid) in the eye in rabbits demonstrated that MNs proved to be efficient in keeping the drug in the eye for 2 months rather than 1 day in other conventional forms due to the capillary drainage system of SCS (Than et al. 2018). In a research study ex,-Vivo MNs were able to deliver $0.02 \mathrm{mg}$ of 6-aminoquinoline into the sclera without clogging the $\mathrm{MN}$ pores due to its micronsize dimensions (Jiang et al. 2009). Glaucoma is an ophthalmic condition associated with the accumulation of aqueous humor resulting in high intraocular pressure and is known to affect nearly 70 million of the population across the world (Weller n.d.). MNs up to size range of 400-700 $\mu \mathrm{m}$ can easily deliver medication in the eye effective upto several months due to the ability of MNs to reduce the production of aqueous humor or decrease the flow of aqueous humor and can deliver the drug as small as $1 \%$ giving similar results to an eye drop (Gupta and Yadav 2019). A study demonstrated intrascleral administration of stainless steel MN loaded with pilocarpine resulted in a 45-fold increase in absorption rate (Jiang et al. 2007). Another study utilized sulforhodamine in conjunction with hollow MNs wherein, the MN array was developed using borosilicate micropipette tubes that limit the quantity of drug administered up to 10-35 $\mu \mathrm{L}$ (Martanto et al. 2006). Figure 4 displays the MN drug delivery to the eye for the treatment of glaucoma.

\subsubsection{Malaria}

Malaria is a disease affecting over 200 million people worldwide each year caused by Plasmodium falciparum causing death (Ali 1989). Artemether (ART) and lumefantrine (LUM) are the standard treatment of choice for malaria but possess certain limitations like hydrophobicity and have low oral bioavailability. Therefore dissolvable MNs were formulated containing the drug in micro-suspension to enhance the bioavailability and demonstration of MN-LUM proved to be more effective in control transdermal delivery over the oral treatment (Pawar and Shende 2020a). MNs array was developed by solvent casting method using artemether co-loaded lumefantrine nanoparticles and the study reported complete permeation of array depicting the drug release upto $60-70 \%$ with stability of 6 months extending the release to $24 \mathrm{~h}$. Thus, the MNs proved to be more efficient and safer in transepidermal delivery in case of BCS class II and IV drugs compared to oral administration (Volpe- Zanutto et al. 2021). The pharmacokinetics study of artemether MN and lumefantrine showed the mean residence time was higher thus, prolongs the release of drug in systemic circulation, improved the bioavailability and proved to be a prominent formulation suitable for combinational antimalarial therapy (Pawar and Shende 2020a, b).

\subsection{MNs in diagnosis}

The conventional blood withdrawal methods are characterized with discomfort and fear among patients, this can be overcome using MNs via its painless biofluids withdrawal method, further the interstitial fluid is extracted from MNs using centrifugation for identification of biomarkers. In addition to blood, urine, saliva, and sweat and interstitial fluids beneath the skin, was discovered as another source of biomarkers for early diagnosis, real-time monitoring, and disease control. MNs present as an unique tool for detecting a wide variety of biomarkers from skin interstitial fluid, including small molecule metabolites, nucleic acids, proteins, and even cells, due to their minimally-invasive nature
Fig. 4 Microneedle drug delivery to eye for treatment of glaucoma

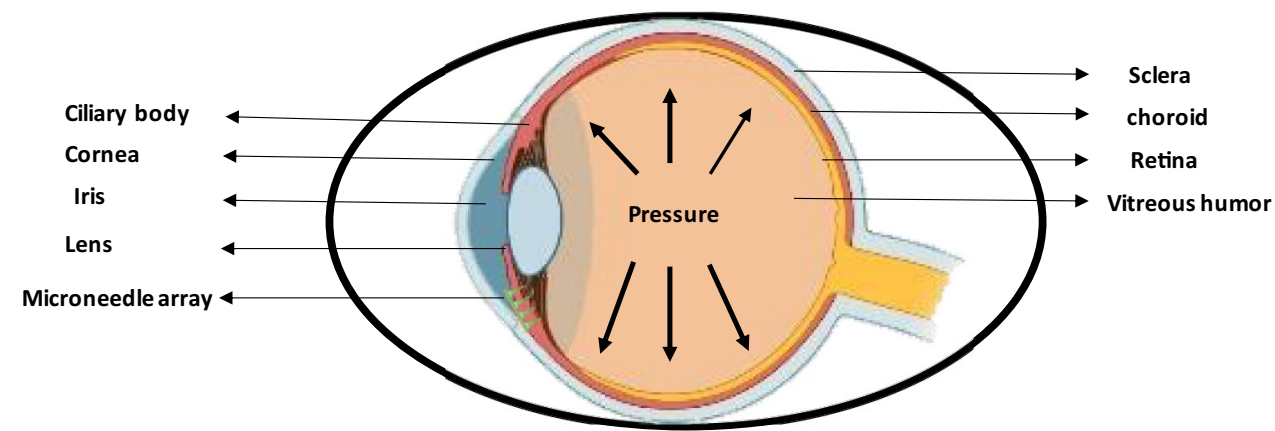


and ease of usage (Zhang et al. 2020). Previous studies showed the status of diseases (cancer, diabetes, arthritis, etc.) by analyzing blood and tissue using hollow MNs or quantum dots (Dugam et al. 2021). A research demonstrated that the silicon MN array are sharp enough to penetrate the stratum corneum and are useful for extraction of transdermal fluid (Lee et al. 2015). Methacrylate hyaluronic acid (MEHA) assisted swellable MNs were developed for rapidly extracting nearly $1.4 \mathrm{mg}$ of ISF for detection of metabolites such as glucose and cholesterol (Chang et al. 2017). Additionally, MNs track immune cells in the skin for example, Poly-l-lysine MN with an alginate hydrogel surface covered with immune adjuvants and antigens was introduced into the skin wherein the leukocytes enter the hydrogel layer and stimulate the adjuvants incorporated in the $\mathrm{MN}$ and thus attract T-cells which are collected for immunological investigation. In another research study hydrophilic hollow MNs were developed for rapid detection of protein biomarkers in interstitial fluid to detect Plasmodium falciparum histidine-rich protein 2, a biomarker for detection of malaria (detected at concentrations as low as $8 \mathrm{mg} / \mathrm{mL}$ ) (Sušić et al. n.d.). MN-based sensors deposited with a pHsensitive iridium oxide layer on to the surface help detect in-vivo concentrations of $\beta$-lactam antibiotics wherein, the sensor detects change in local $\mathrm{pH}$ caused due to hydrolysis of lactam ring by lactamase enzyme immobilized on the electrode surface and these biosensors are sterilizable and stable for up to two weeks at $20{ }^{\circ} \mathrm{C}$ (Gowers et al. 2019). Another research study demonstrated that conventional MNs if inserted for a long time in humans cause risk of $\mathrm{MN}$ breakage induced by stress or motion and thus, the researches fabricated and evaluated porous PDMS matrix $\mathrm{MN}$ with a coating of hyaluronic acid. Furthermore, they proved that flexible MNs are successful in extracting ISF in-vivo (mouse) and in-vitro by compression for continuous glucose monitoring (Takeuchi et al. 2020).

\section{Pre-clinical and clinical studies of $\mathrm{MN}$-based theranostics}

A lot of research is carried out on the treatment and diagnosis of diseases using MNs and currently, there are about 64 clinical trial studies on MN. A clinal trial conducted on the delivery of insulin using MNs in the treatment of diabetes type 1 in 2009 and was completed (Koschinsky and Heinemann 2001). Glucose measurement with a MN is another clinical trial study conducted in February 2016 (Study 2021). There is no clinical trial conducted on the theranostics of MNs. So, this is the future challenge for the researchers for the growth of the pharmaceutical industry in developing MNs for theranostics.

\section{Regulatory requirements}

In the pharmaceutical industry, MN systems are relatively modern technology and as stated before, there are four different types of MN systems that are manufactured employing a range of technologies. As a result, standardized rules for the use of established techniques for production, evaluation, test criteria for approval, and quality control are critical for industries and researchers. Therefore, FDA issued "Regulatory Considerations for Microneedling Devices" and "Scientific Considerations for MN Products" in 2017 to aid in the development of MNs as a medical device. According to Sect. 201 (h) of the Federal Food, Drug, and Cosmetic Act (Aut 2021). MNs are devices intended to cure or monitor a particular disease or condition. MNs possess an array of sharp tips like hypodermic needles and comes under class II device under 21 CFR 878. 4430. These products are considered as medical devices and are regulated by the CDRH (Center for devices and radiological health) or the combination product category, regulated by either CDER (Center for drug evaluation and research) or CBER (Center for biological evaluation and research) divisions of the US FDA. Device components, technical description of device like needle geometry and insertion depth, suggested course of treatment, disposal instructions, processing instructions for reusable products, and expiry must include on the labelling for these types of devices (FDA 2020). Regulatory authorities certainly need comprehensive studies into metabolism and elimination routes, potentially delaying commercialization. Unlike conventional transdermal and topical drug delivery systems, MN comes in contact with viable skin cells, hence regulators require $\mathrm{MN}$ systems to be sterile (Larrañeta et al. 2016a, b).

\section{Conclusions}

MNs are unique because of their diverse qualities and characteristics, despite the fact that different drug delivery systems are being launched on a nanoscale for the targeted distribution of active compounds. MN technology will aid in the creation of new and improved devices with a wide range of biomedical and other applications that are smaller, cheaper, pain-free, and more convenient. In order to further boost drug permeability, a combination of ultrasound and transdermal drug administration is being investigated. As a result, MNs are made with a number of alterations in order to distribute drugs effectively through the skin, paving the way for a new era in transdermal drug delivery systems. The cold chain can be avoided since most MNs formulate biomolecules in a dry state. As a result, MN technology has the potential to dramatically 
increase vaccination delivery in underdeveloped countries. Future research must be targeted towards utilizing mics for theranostic purposes which will play a critical role in advancing clinical applications and novel research for pharmaceutical scientists and researchers.

Funding Not applicable.

\section{Declarations}

Conflict of interests The authors declared no conflict of interest concerning with this article.

\section{References}

L. Agrawal, S.K. Vimal, M.H. Chen, T. Shiga, An Idea of Using Microneedles for the Targeted Drug Delivery to Overcome the Blood Brain Barrier for the Treatment of Brain Diseases. J. Pharmacovigil. 06(05), 1-4 (2018). https://doi.org/10.4172/2329-6887. 1000270

Z. Ali, Malaria 1-21 (1989)

Aut Global Health, For Microneedling 1-5 (2021)

S. Babity, M. Roohnikan, D. Brambilla, Advances in the Design of Transdermal Microneedles for Diagnostic and Monitoring Applications 14(49), 1-16 (2018). https://doi.org/10.1002/smll.201803186.

S.H. Bariya, M.C. Gohel, T.A. Mehta, O.P. Sharma, Microneedles: An Emerging Transdermal Drug Delivery System. J. Pharm. Pharmacol. 64(1), 11-29 (2012). https://doi.org/10.1111/j.2042-7158. 2011.01369.x

B.W. Barry, Novel Mechanisms and Devices to Enable Successful Transdermal Drug Delivery. Eur. J. Pharm. Sci. 14(2), 101-114 (2001). https://doi.org/10.1016/S0928-0987(01)00167-1

S. Bhatnagar, P.R. Gadeela, P. Thathireddy, V.V.K. Venuganti, Microneedle-Based Drug Delivery: Materials of Construction. J. Chem. Sci. 131(9), (2019). https://doi.org/10.1007/s12039-019-1666-x.

J. Bullock, · Rizvi S.A.A. · Saleh A.M. · Ahmed S.S. · Do D.P. · Ansari R.A. Ahmed., Rheumatoid Arthritis : A Brief Overview of the Treatment. Medical Principles and Practice Mini 27, 501-507 (2018)

J. Cao, Su. Jingjing, M. An, Y. Yang, Yi. Zhang, J. Zuo, N. Zhang, Y. Zhao, Novel DEK-Targeting Aptamer Delivered by a Hydrogel Microneedle Attenuates Collagen-Induced Arthritis. Mol. Pharm. 18(1), 305-316 (2021). https://doi.org/10.1021/acs.molpharmaceut. 0c00954

J. Cao, N. Zhang, Z. Wang, Su. Jingjing, J. Yang, J. Han, Y. Zhao, Microneedle-Assisted Transdermal Delivery of Etanercept for Rheumatoid Arthritis Treatment. Pharmaceutics 11(5), 1-12 (2019). https://doi.org/10.3390/pharmaceutics11050235

Á. Cárcamo-Martínez, B. Mallon, J. Domínguez-Robles, L.K. Vora, Q.K. Anjani, R.F. Donnelly, Hollow Microneedles: A Perspective in Biomedical Applications. Int. J. Pharm. 599, 1-3 (2021). https://doi.org/10.1016/j.ijpharm.2021.120455

A. Cárcamo-Martínez, B. Mallon, J. Domínguez-Robles, L.K. Vora, Q.K. Anjani, R.F. Donnelly, Hollow Microneedles : A Perspective Biomed. Appl. 1-3 (2021)

H. Chang, M. Zheng, Yu. Xiaojun, A. Than, R.Z. Seeni, R. Kang, J. Tian et al., A Swellable Microneedle Patch to Rapidly Extract Skin Interstitial Fluid for Timely Metabolic Analysis. Adv. Mater. 29(37), 1-8 (2017). https://doi.org/10.1002/adma.201702243
C. Weller, Microneedles For Glaucoma Deliver Drugs Straight Into The Eye, Focusing Treatment. (n.d.)

M. Cormier, B. Johnson, M. Ameri, K. Nyam, L. Libiran, D.D. Zhang, P. Daddona, Transdermal Delivery of Desmopressin Using a Coated Microneedle Array Patch System. J. Control. Release 97(3), 503-511 (2004). https://doi.org/10.1016/j.jconrel.2004. 04.003

M. Dangol, S. Kim, C.G. Li, S.F. Lahiji, M. Jang, Y. Ma, I. Huh, H. Jung, Anti-Obesity Effect of a Novel Caffeine-Loaded Dissolving Microneedle Patch in High-Fat Diet-Induced Obese C57BL/6J Mice. J. Control. Release 265, 41-47 (2017). https://doi.org/10. 1016/j.jconrel.2017.03.400

P. Dardano, M. Battisti, S. De Martino, I. Rea, B. Miranda, L. Nicolais, L. De Stefano, Theranostic Microneedle Devices: Innovative Biosensing and Transdermal Drugs Administration Biosensors Curr. Novel Strat. Biosens. 1-29 (2021). https://doi.org/10.5772/ intechopen. 95050

P. Dardano, A. Caliò, V. Di Palma, M.F. Bevilacqua, A.D. Di Matteo, L. Stefano, A Photolithographic Approach to Polymeric Microneedles Array Fabrication. Materials (2015). https://doi.org/10.3390/ ma8125484

R.F. Donnelly, T.R.R. Singh, A. David Woolfson, Microneedle-Based Drug Delivery Systems: Microfabrication, Drug Delivery, and Safety. Drug Delivery 17(4), 187-207 (2010). https://doi.org/10. 3109/10717541003667798

R.F. Donnelly, T.R.R. Singh, M.J. Garland, K. Migalska, R. Majithiya, C.M. McCrudden, P.L. Kole, T.M.T. Mahmood, H.O. McCarthy, A. David Woolfson, Hydrogel-Forming Microneedle Arrays for Enhanced Transdermal Drug Delivery. Adv. Func. Mater. 22(23), 4879-4890 (2012). https://doi.org/10.1002/adfm.201200864

S. Dugam, R. Tade, R. Dhole, S. Nangare, Emerging Era of Microneedle Array for Pharmaceutical and Biomedical Applications: Recent Advances and Toxicological Perspectives. Future Journal of Pharmaceutical Sciences 7(1), 1-26 (2021). https://doi.org/10. 1186/s43094-020-00176-1

FDA, Regulatory Considerations for Microneedling Products Guidance for Industry and Food and Drug Administration Staff. 301, $1-12(2020)$

S.A.N. Gowers, D.M.E. Freeman, T.M. Rawson, M.L. Rogers, R.C. Wilson, A.H. Holmes, A.E. Cass, D. O'Hare, Development of a Minimally Invasive Microneedle-Based Sensor for Continuous Monitoring of $\beta$-Lactam Antibiotic Concentrations in Vivo. ACS Sensors 4(4), 1072-1080 (2019). https://doi.org/10.1021/ acssensors.9b00288

A.J. Guillot, A.S. Cordeiro, R.F. Donnelly, M. Carmen Montesinos, T.M. Garrigues, A. Melero, Microneedle-based Delivery: An Overview of Current Applications and Trends. Pharmaceutics 12(6), 1-28 (2020). https://doi.org/10.3390/pharmaceutics 12060569

J. Gupta, E.I. Felner, M.R. Prausnitz, Minimally Invasive Insulin Delivery in Subjects with Type 1 Diabetes Using Hollow Microneedles. Diabetes Technol. Ther. 11(6), 329-37 (n.d.)

J. Gupta, D.D. Denson, E.I. Felner, M.R. Prausnitz, Rapid Local Anesthesia in Humans Using Minimally Invasive Microneedles. Clin. J. Pain 28(2), 129-135 (2012). https://doi.org/10.1097/AJP. 0b013e318225dbe9

P. Gupta, K.S. Yadav, Applications of Microneedles in Delivering Drugs for Various Ocular Diseases. Life Sci. 237, (2019). https:// doi.org/10.1016/j.1fs.2019.116907.

J. Halder, S. Gupta, R. Kumari, G.D. Gupta, V.K. Rai, Microneedle Array: Applications, Recent Advances, and Clinical Pertinence in Transdermal Drug Delivery. J. Pharm. Innov. (2020). https:// doi.org/10.1007/s12247-020-09460-2

M.T. Hoang, K.B. Ita, D.A. Bair, Solid Microneedles for Transdermal Delivery of Amantadine Hydrochloride and Pramipexole Dihydrochloride. Pharmaceutics 7(4), 379-396 (2015). https:// doi.org/10.3390/pharmaceutics7040379 
J. Jiang, H.S. Gill, D. Ghate, B.E. McCarey, S.R. Patel, H.F. Edelhauser, M.R. Prausnitz, Coated Microneedles for Drug Delivery to the Eye. Invest. Ophthalmol. vis. Sci. 48(9), 4038-4043 (2007). https://doi. org/10.1167/iovs.07-0066

J. Jiang, J.S. Moore, H.F. Edelhauser, M.R. Prausnitz, Intrascleral Drug Delivery to the Eye Using Hollow Microneedles. Pharm. Res. 26(2), 395-403 (2009). https://doi.org/10.1007/ s11095-008-9756-3

H. Jun, M.R. Han, N.G. Kang, J.H. Park, J.H. Park, Use of Hollow Microneedles for Targeted Delivery of Phenylephrine to Treat Fecal Incontinence. J. Control. Release 207(Cdc), 1-6 (2015). https://doi.org/10.1016/j.jconrel.2015.03.031.

M.C. Kearney, E. Caffarel-Salvador, S.J. Fallows, H.O. McCarthy, R.F. Donnelly, Microneedle-Mediated Delivery of Donepezil: Potential for Improved Treatment Options in Alzheimer's Disease. Eur. J. Pharm. Biopharm. 103, 43-50 (2016). https://doi. org/10.1016/j.ejpb.2016.03.026.

N. Khare, P. Shende, Microneedle System: A Modulated Approach for Penetration Enhancement. Drug Dev. Ind. Pharm. 1-10 (2021) https://doi.org/10.1080/03639045.2021.1992421

E. Kim, G. Erdos, S. Huang, T.W. Kenniston, S.C. Balmert, C.D. Carey, V. Stalin Raj et al., Microneedle Array Delivered Recombinant Coronavirus Vaccines: Immunogenicity and Rapid Translational Development. EBioMedicine 55, 1-12 (2020). https:// doi.org/10.1016/j.ebiom.2020.102743

J.Y. Kim, M.R. Han, Y.H. Kim, S.W. Shin, S.Y. Nam, J.H. Park, TipLoaded Dissolving Microneedles for Transdermal Delivery of Donepezil Hydrochloride for Treatment of Alzheimer's Disease. Eur. J. Pharm. Biopharm. 105, 148-155 (2016). https://doi.org/ 10.1016/j.ejpb.2016.06.006

T. Koschinsky, L. Heinemann, Sensors for Glucose Monitoring: Technical and Clinical Aspects. Diabetes Metab. Res. Rev. 17(2), 113-123 (2001). https://doi.org/10.1002/dmrr.188

K.J. Krieger, N. Bertollo, M. Dangol, J.T. Sheridan, M.M. Lowery, E.D. O'Cearbhaill, Simple and Customizable Method for Fabrication of High-Aspect Ratio Microneedle Molds Using Low-Cost 3D Printing. Microsyst. Nanoeng. 5(1), 1-39 (2019). https://doi. org/10.1038/s41378-019-0088-8

E. Larrañeta, R.E.M. Lutton, A. David Woolfson, R.F. Donnelly, Microneedle Arrays as Transdermal and Intradermal Drug Delivery Systems: Materials Science, Manufacture and Commercial Development. Mater. Sci. Eng. R. Rep. 104, 1-32 (2016a). https://doi.org/10.1016/j.mser.2016.03.001

E. Larrañeta, M.T.C. McCrudden, A.J. Courtenay, R.F. Donnelly, Microneedles: A New Frontier in Nanomedicine Delivery. Pharm. Res. 33(5), 1055-1073 (2016b). https://doi.org/10.1007/ s11095-016-1885-5

H.J. Lee, Y. Son, D. Kim, Y.K. Kim, N. Choi, E.S. Yoon, I.J. Cho, A New Thin Silicon Microneedle with an Embedded Microchannel for Deep Brain Drug Infusion. Sens. Actuators, B Chem. 209, 413-422 (2015). https://doi.org/10.1016/j.snb.2014.11.132

K.J. Lee, S.S. Jeong, D.H. Roh, D.Y. Kim, H.K. Choi, E.H. Lee, A Practical Guide to the Development of Microneedle Systems - In Clinical Trials or on the Market. Int. J. Pharm. 573, (2020). https:// doi.org/10.1016/j.ijpharm.2019.118778

Y. Levin, E. Kochba, I. Hung, R. Kenney, Intradermal Vaccination Using the Novel Microneedle Device MicronJet600: Past, Present, and Future. Hum. Vaccin. Immunother. 11(4), 991-997 (2015). https://doi.org/10.1080/21645515.2015.1010871

G.S. Liu, Y. Kong, Y. Wang, Y. Luo, X. Fan, Xi. Xie, B.R. Yang, XWu. Mei, Microneedles for Transdermal Diagnostics: Recent Advances and New Horizons. Biomaterials 232, 1-10 (2020). https://doi. org/10.1016/j.biomaterials.2019.119740

K. Van Der Maaden, W. Jiskoot, J. Bouwstra, Microneedle Technologies for (Trans)Dermal Drug and Vaccine Delivery. J. Control. Release
161(2), 645-655 (2012). https://doi.org/10.1016/j.jconrel.2012.01. 042

K. Van Der Maaden, S.J. Trietsch, H. Kraan, E.M. Varypataki, S. Romeijn, R. Zwier, H.J. Van Der Linden et al., Novel Hollow Microneedle Technology for Depth-Controlled MicroinjectionMediated Dermal Vaccination: A Study with Polio Vaccine in Rats. Pharm. Res. 31(7), 1846-1854 (2014). https://doi.org/10. 1007/s11095-013-1288-9

W. Martanto, J.S. Moore, O. Kashlan, R. Kamath, P.M. Wang, J.M. O'Neal, M.R. Prausnitz, Microinfusion Using Hollow Microneedles. Pharm. Res. 23(1), 104-113 (2006). https://doi.org/10.1007/ s11095-005-8498-8

A.F. Moreira, C.F. Rodrigues, T.A. Jacinto, S.P. Miguel, E.C. Costa, I.J. Correia, Microneedle-Based Delivery Devices for Cancer Therapy: A Review. Pharmacol. Res. 148, (2019). https://doi.org/ 10.1016/j.phrs.2019.104438.

T.T. Nguyen, V. Van Giau, T.K. Vo, Current Advances in Transdermal Delivery of Drugs for Alzheimer's Disease. Indian Journal of Pharmacology 49(2), 145-154 (2017). https://doi.org/10.4103/ 0253-7613.208143

S. Pawar, P. Shende, 22 Factorial Design-Based Biocompatible Microneedle Arrays Containing Artemether Co-Loaded With Lumefantrine Nanoparticles for Transepidermal Delivery. Biomed. Microdevice 22(1), 1-15 (2020a). https://doi.org/10. 1007/s10544-020-0476-8

S. Pawar, P. Shende, A Comparative Outlook on Pharmacokinetics and Antimalarial Studies of Artemether and Lumefantrine-Loaded Microneedle Patches and a Dry Suspension Containing Nanosponges. J. Drug Deliv. Sci. Technol. 60, (2020b). https://doi.org/ 10.1016/j.jddst.2020.102055.

V. Rastogi, P. Yadav, Transdermal Drug Delivery System: An Overview. Asian Journal of Pharmaceutics 6(3), 161-170 (2012). https://doi.org/10.4103/0973-8398.104828

D. Sharma, Microneedles : An Approach in Transdermal Drug Delivery : A Review. Pharma Tutor 1-26 (2018)

P. Shende, M. Salunke, Transepidermal Microneedles for CoAdministration of Folic Acid with Methotrexate in the Treatment of Rheumatoid Arthritis. Biomed. Phys.Eng. Express 5(2), (2019). https://doi.org/10.1088/2057-1976/aafbbb.

T.R. Singh, H.M. Raj, K. Mooney, A.Z. Alkilani, R.F. Donnelly, Microneedles for Drug Delivery and Monitoring. Microfluidic Devices for Biomedical Applications (2013). https://doi.org/10. 1533/9780857097040.2.185

Study Previous, Microneedle Glucose Measurement Using Microneedle Patches ( GUMP ). no. Cdc, 2-7 (2021)

A. Sušić, Z. Hrnjica, I. Kajgana, M. Mujezinović, A. Hasanbegović, J. Brčkalo, A. Tucak, M. Sirbubalo, E. Vranić, Use of Hollow Microneedle Drug Delivery Systems in Treatment of Diabetes Mellitus. Int. Conf. Med. Biol. Eng. 575-80 (n.d.)

N. Tabassum, A. Sofi, T. Khuroo, Microneedle Technology: A New Drug Delivery System. Int. J. Res. Pharm. Biomed. Sci 2(1), 59-62 (2011)

K. Takeuchi, N. Takama, R. Kinoshita, T. Okitsu, B. Kim, Flexible and Porous Microneedles of PDMS for Continuous Glucose Monitoring. Biomed. Microdevice 22(4), 1-12 (2020). https://doi.org/10. 1007/s10544-020-00532-1

T. Singh, R. Raj, I. Tekko, K. McAvoy, H. McMillan, D. Jones, R.F. Donnelly, Minimally Invasive Microneedles for Ocular Drug Delivery. Expert Opin. Drug Deliv. 14(4), 525-537 (2017). https://doi.org/10.1080/17425247.2016.1218460

A. Than, C. Liu, H. Chang, P.K. Duong, C.M.G. Cheung, Xu. Chenjie, X. Wang, P. Chen, Self-Implantable Double-Layered MicroDrug-Reservoirs for Efficient and Controlled Ocular Drug Delivery. Nat. Commun. 9(1), 1-12 (2018). https://doi.org/10.1038/ s41467-018-06981-w 
T.M. Tuan-Mahmood, M.T.C. McCrudden, B.M. Torrisi, E. McAlister, M.J. Garland, T.R.R. Singh, R.F. Donnelly, Microneedles for Intradermal and Transdermal Drug Delivery. Eur. J. Pharm. Sci. 50(5), 623-637 (2013). https://doi.org/10.1016/j.ejps.2013.05.005

A. Tucak, M. Sirbubalo, L. Hindija, O. Rahić, J. Hadžiabdić, K. Muhamedagić, A. Čekić, E. Vranić, Microneedles: Characteristics, Materials, Production Methods and Commercial Development. Micromachines 11(11), 1-30 (2020). https://doi.org/10. 3390/mi11110961

V.K. Venuganti, S. Bhatnagar, N.G. Bankar, M.V. Kulkarni, V. Vamsi, K. Venuganti, Dissolvable Microneedle Patch Containing Doxorubicin and Docetaxel Is Effective in 4T1 Xenografted Breast Cancer Mouse Model. International Journal of Pharmaceutics. Int. J. Pharm. 556, 263-75 (n.d.)

F. Volpe-Zanutto, L.T. Ferreira, A.D. Permana, M. Kirkby, A.J. Paredes, L.K. Vora, A.P. Bonfanti et al., Artemether and Lumefantrine Dissolving Microneedle Patches with Improved Pharmacokinetic Performance and Antimalarial Efficacy in Mice Infected with Plasmodium Yoelii. J. Control. Release 333, 298-315 (2021). https://doi.org/10. 1016/j.jconrel.2021.03.036

M. Waghchaure, S. Govardhane, P. Shende, Enhancement of Immunopotentiation Using Tetanus Toxoid-Based Nanoparticulate Dissolvable Microneedles. Biomed. Microdevice 23(3), 2-10 (2021). https://doi.org/10.1007/s10544-021-00571-2

T. Waghule, G. Singhvi, S.K. Dubey, M.M. Pandey, G. Gupta, M. Singh, K. Dua, Microneedles_A Smart Approach and Increasing Potential for Transdermal Drug Delivery System. In 109, 1249-58 (n.d.)

G. Widera, J. Johnson, L. Kim, L. Libiran, K. Nyam, P.E. Daddona, M. Cormier, Effect of Delivery Parameters on Immunization to Ovalbumin Following Intracutaneous Administration by a Coated Microneedle Array Patch System. Vaccine 24(10), 1653-1664 (2006). https://doi.org/10.1016/j.vaccine.2005.09.049

H.L. Wong, N. Chattopadhyay, X.Y. Wu, R. Bendayan, Nanotechnology Applications for Improved Delivery of Antiretroviral Drugs to the Brain. Adv. Drug Deliv. Rev. 62(4-5), 503-517 (2010). https://doi.org/10.1016/j.addr.2009.11.020

L. Xie, H. Zeng, J. Sun, W. Qian, Engineering Microneedles for Therapy and Diagnosis: A Survey. Micromachines 11(3), 1-28 (2020). https://doi.org/10.3390/mi11030271

Xi. Xie, C. Pascual, C. Lieu, Oh. Seajin, Ji. Wang, B. Zou, J. Xie et al., Analgesic Microneedle Patch for Neuropathic Pain Therapy. ACS Nano 11(1), 395-406 (2017). https://doi.org/10.1021/acsnano. $6 \mathrm{~b} 06104$

J. Yang, X. Liu, Fu. Yunzhi, Y. Song, Recent Advances of Microneedles for Biomedical Applications: Drug Delivery and Beyond.
Acta Pharmaceutica Sinica B 9(3), 469-483 (2019). https://doi. org/10.1016/j.apsb.2019.03.007

Q. Yang, W. Zhong, L. Xu, H. Li, Q. Yan, Y. She, G. Yang, Recent Progress of 3D-Printed Microneedles for Transdermal Drug Delivery. Int. J. Pharm. 593, Elsevier B.V. (2021). https://doi.org/10.1016/j. ijpharm.2020.120106.

J. Yu, Y. Zhang, Y. Ye, R. DiSanto, W. Sun, D. Ranson, F.S. Ligler, J.B. Buse, Z. Gu, Microneedle-Array Patches Loaded with HypoxiaSensitive Vesicles Provide Fast Glucose-Responsive Insulin Delivery. Diabetes Technol. Ther. 18, S54-55 (2016). https://doi. org/10.1089/dia.2016.2505

M. Zaric, O. Lyubomska, O. Touzelet, C. Poux, S. Al-Zahrani, F. Fay, L. Wallace et al., Skin Dendritic Cell Targeting via Microneedle Arrays Laden with Antigen-Encapsulated Poly- D, 1 -Lactide- Co -Glycolide Nanoparticles Induces Efficient Antitumor and Antiviral Immune Responses. ACS Nano 7(3), 2042-2055 (2013). https://doi.org/10.1021/nn304235j

X. Zhang, Y. Wang, J. Chi, Y. Zhao, Smart Microneedles for Therapy and Diagnosis. Research 2020, 1-26 (2020). https://doi.org/10. 34133/2020/7462915.

Y. Zhang, K. Brown, K. Siebenaler, A. Determan, D. Dohmeier, K. Hansen, Development of Lidocaine-Coated Microneedle Product for Rapid, Safe, and Prolonged Local Analgesic Action. Pharm. Res. 29(1), 170-177 (2012). https://doi.org/10.1007/ s11095-011-0524-4

Y. Zhang, Q. Liu, Yu. Jicheng, Yu. Shuangjiang, J. Wang, Li. Qiang, Gu. Zhen, Locally Induced Adipose Tissue Browning by Microneedle Patch for Obesity Treatment. ACS Nano 11(9), 9223-9230 (2017). https://doi.org/10.1021/acsnano.7b04348

Y. Zhang, Yu. Jicheng, Di. Wen, G. Chen, Gu. Zhen, The Potential of a Microneedle Patch for Reducing Obesity. Expert Opin. Drug Deliv. 15(5), 431-433 (2018). https://doi.org/10.1080/17425247. 2018.1449831

G. Zheng, K. Sayama, T. Okubo, L.R. Juneja, I. Oguni, Anti-Obesity Effects of Three Major Components of Green Tea, Catechins, Caffeine and Theanine, in Mice. In Vivo 18(1), 55-62 (2004)

C.P. Zhou, Y.L. Liu, H.L. Wang, P.X. Zhang, J.L. Zhang, Transdermal Delivery of Insulin Using Microneedle Rollers in Vivo. Int. J. Pharm. 392(1-2), 127-133 (2010). https://doi.org/10.1016/j.ijpharm.2010. 03.041

Publisher's Note Springer Nature remains neutral with regard to jurisdictional claims in published maps and institutional affiliations. 